\title{
Tolerance of Bermudagrass to Herbicides
}

\section{R. W. BOVEY, R. E. MEYER, AND E. C. HOLT}

Highlight: Herbicides 2,4-D, 2,4,5-T and dicamba applied in spring or fall usually did not reduce yields of bermudagrass. When applied during dry periods, picloram reduced density and yield of bermudagrass. Degree of bermudagrass injury was directly related to rate of herbicide. "Common," "Coastal," and "Coastcross-1" varieties responded similarly to each herbicide studied. Kleingrass, a new forage grass growing in the plot area, was tolerant of all herbicide treatments, including picloram.

In south Texas, many rangeland areas that have been disturbed and established in bermudagrass (Cynodon dactylon (L.) Pers.) pastures are now rapidly becoming infested with seedlings of huisache (Acacia farnesiana (L.) Willd.) and honey mesquite (Prosopis juliflora (Swartz) DC. var. glandulosa (Torr.) Cockerell). Seed of these woody species are abundant on some sites. They germinate and rapidly establish seedlings under favorable conditions. Within 2 or 3 years, the woody plants, especially huisache, may become too large to mow. The field must then either be abandoned or treated by other, more drastic mechanical methods. Annual mowing will not kill the brush, and huisache is resistant to broadcast sprays of 2,4-D [(2,4-dichlorophenoxy)acetic acid] or 2,4,5-T [2,4,5-trichlorophenoxy)acetic acid].

Huisache and honey mesquite are susceptible to picloram (4-amino-3,5,6trichloropicolinic acid) and dicamba (3,6-dichloro-o-anisic acid) (Bovey et al., 1969). Combinations of picloram + dicamba or 2,4,5-T are effective against both species, and dicamba $+2,4,5-\mathrm{T}$ will control honey mesquite (Meyer and

Authors are agronomist and plant physiologist, U.S. Department of Agriculture, Agricultural Research Service, Department of Range Science, and professor, Department of Soil and Crop Sciences, Texas A\&M University, College Station.

The study is a contribution from U.S. Dep. Agr., Agr. Res. Serv. and the Texas Agricultural Experiment Station, College Station. This paper does not constitute a recommendation of this product by the U.S. Dep. Agr.

Manuscript received July 5, 1973.
Bovey, 1973; Scifres and Hoffman, 1972). Apparently, 2,4-D, 2,4,5-T, tolyl)oxy] propionic acid) and certain combinations of these materials applied for herbaceous weed control did not damage bermudagrass turf at College Station, Texas (McBee, 1966). Reber et al. (1971) found that several bermudagrass cultivars in Illinois were susceptible to picloram; resistant cultivars recovered within 16 months following treatment.

The relative tolerance of "Common," "Coastal," and "Coastcross-1" varieties of bermudagrass to 2,4-D, 2,4,5-T, dicamba, and picloram treated in spring and fall with rates commonly used for herbaceous and woody plant control was evaluated in this study.

\section{Materials and Methods}

Granule or spray formulations of the potassium salt of picloram and the dimethylamine salt of dicamba were applied to triplicate plots of 5 by $20 \mathrm{ft}$ in established stands of bermudagrass near College Station, Texas. A randomized block or split-plot design was used. Soil was a Lufkin sandy loam. Sprays of the propylene glycol butyl ether esters of 2,4-D and 2,4,5-T or butoxyethanol ester of 2,4,5-T were also included. Spray formulations were applied in water by a hand-carried boom at 20 gallons per acre. Karbutilate [tert-butylcarbamic acid ester with 3-( $m$-hydroxyphenyl)-1,1-dimethylurea] granules were applied on May 15, 1972.

Active ingredient of picloram, dicamba, and karbutilate granules were 2 , dicamba, MCPP (2-[(4-chloro-o-
10 , and $10 \%$, respectively. Herbicides were applied on October 30, 1970; February 17,1971 ; May 3, 1971; October 14, 1971; and May 15, 1972. Bermudagrass was harvested in the spring and fall after herbicide treatment. Forage from a 2.8 by 10 -foot area was collected from the center of each plot. Oven-dry weights were obtained, and forage yields were converted to $\mathrm{lb} /$ acre. Herbicides and rates used are given in the tables that follow.

\section{Results}

Picloram granules applied in October 1970 at 2 and $4 \mathrm{lb} /$ acre significantly reduced yields of Coastal and Coastcross1 bermudagrass when harvested in June and October 1971 (Table 1). Most of the grass harvested in plots treated with picloram at $4 \mathrm{lb} / \mathrm{acre}$ was Kleingrass (Panicum coloratum L.), which appeared very tolerant of all picloram treatments. Kleingrass is a recent forage plant introduction. A thin stand of Kleingrass volunteered throughout the plot area from a previous planting and appeared to increase in picloram-treated plots.

Picloram granules applied to established Common bermudagrass in October, 1970, produced yields similar to those obtained for Coastal and Coastcross-1 harvested in June, 1971. Picloram at 1, 2 and $4 \mathrm{lb} / \mathrm{acre}$ caused yields of 1647,666 and $86 \mathrm{lb} / \mathrm{acre}$, oven-dry forage, respectively, compared to the control of 2918 $\mathrm{lb} /$ acre (data not shown). Rainfall was below normal after treatment (Table 2), and phytotoxic levels of picloram were probably not leached from the root zone. Dicamba granules applied at 1, 2 or 4 $\mathrm{lb} / \mathrm{acre}$ in October, 1970, did not reduce yield of Common bermudagrass, which produced about $3500 \mathrm{lb}$ oven-dry forage/ acre, by June, 1971. The control produced $2918 \mathrm{lb} /$ acre oven-dry forage (data not shown). Yield of Coastal bermuda- 
Table 1. Oven-dry weight (lb/acre) of vegetation on Coastal and Coastcross-1 bermudagrass sites at College Station, Texas, after application of picloram and dicamba granules on October 30, 1970, and harvested at two dates.

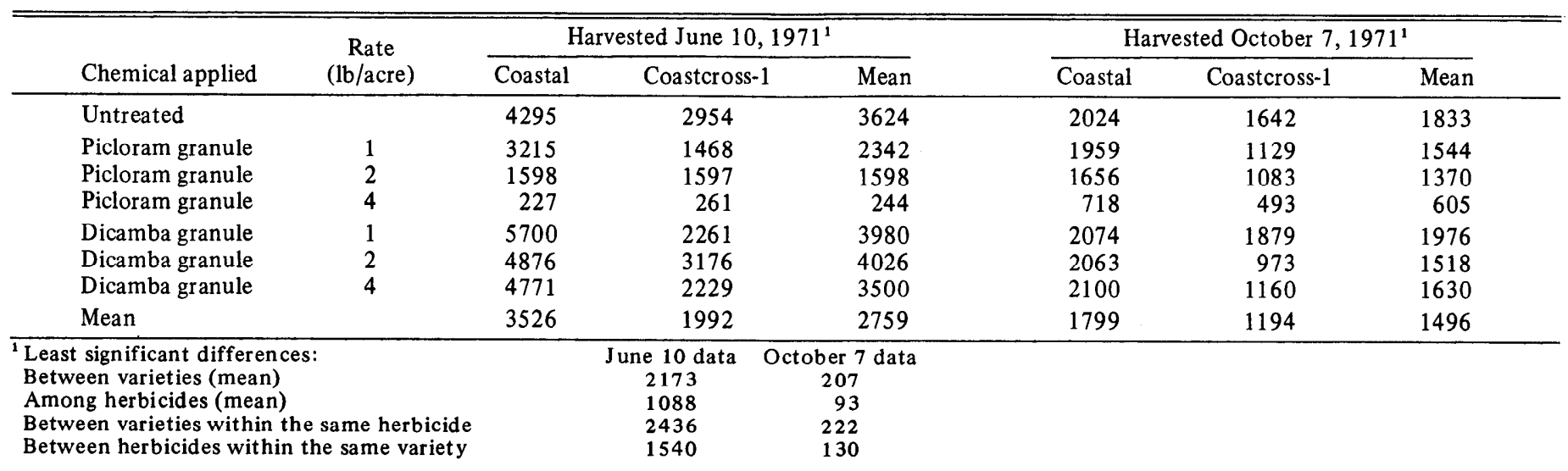

grass was unaffected in spring and fall harvests, , but yield of Coastcross-1 was reduced in the fall harvest in plots receiving dicamba at 2 and $4 \mathrm{lb} / \mathrm{acre}$ (Table 1 ).

Picloram granules at 1,2 and $4 \mathrm{lb} / \mathrm{acre}$ applied to plots in February, 1971, significantly reduced yields of Coastal and Coastcross-1 bermudagrass by June 1971 (data not shown). However, grass in the same plots harvested in October, 1971, had about recovered. Significant reduction in yield occurred only in plots receiving $4 \mathrm{lb} /$ acre of picloram. Dicamba granules did not reduce forage yields on Coastal or Coastcross-1 varieties from either spring or fall harvests. Drought conditions prevailed during the winter of $1970-1971$, and injury to the bermudagrass from picloram may have been accentuated since rainfall is important in dissipation of the herbicide from the soil profile. Yield data for Common bermudagrass from plots treated in February, 1971, were similar to plots treated in October, 1970.

Spring applications (May, 1971) of picloram sprays and granules reduced forage growth of Common bermudagrass at most rates when yields were taken 1
Table 2. Rainfall (inches) between treatment date and harvest date at College Station, Texas.

\begin{tabular}{ccc}
\hline $\begin{array}{c}\text { Treatment } \\
\text { date }\end{array}$ & $\begin{array}{c}\text { Harvest } \\
\text { date }\end{array}$ & $\begin{array}{c}\text { Accumulative } \\
\text { rainfall }\end{array}$ \\
\hline $10 / 30 / 70$ & $6 / 10 / 71$ & 11.65 \\
& $10 / 7 / 71$ & 22.58 \\
$1 / 17 / 71$ & $6 / 10 / 71$ & 9.30 \\
& $10 / 7 / 71$ & 20.23 \\
$5 / 3 / 71$ & $6 / 10 / 71$ & 6.13 \\
& $10 / 7 / 71$ & 17.06 \\
$10 / 14 / 71$ & $6 / 20 / 72$ & 21.65 \\
& $11 / 9 / 72$ & 39.04 \\
$5 / 15 / 72$ & $6 / 20 / 72$ & 1.06 \\
& $11 / 9 / 72$ & 18.45 \\
\hline
\end{tabular}

Table 3. Oven-dry weight (lb/acre) of vegetation on Coastal and Coastcross-1 bermudagrass sites at College Station, Texas, after application of herbicide sprays and granules on May 3, 1971, and harvested at two dates.

\begin{tabular}{|c|c|c|c|c|c|c|c|}
\hline \multirow[b]{2}{*}{ Chemical applied } & \multirow{2}{*}{$\begin{array}{c}\text { Rate } \\
\text { (lb/acre) }\end{array}$} & \multicolumn{3}{|c|}{ Harvested June $10,1971^{1}$} & \multicolumn{3}{|c|}{ Harvested October $7,1971^{1}$} \\
\hline & & Coastal & Coastcross- 1 & Mean & Coastal & Coastcross-1 & Mean \\
\hline Untreated & & 6430 & 2979 & 4704 & 2498 & 1748 & 2123 \\
\hline $\begin{array}{l}\text { Picloram spray } \\
\text { Picloram spray } \\
\text { Picloram spray } \\
\text { Picloram spray }\end{array}$ & $\begin{array}{l}0.5 \\
1 \\
2 \\
4\end{array}$ & $\begin{array}{l}3342 \\
1988 \\
2409 \\
2081\end{array}$ & $\begin{array}{r}1008 \\
1021 \\
1113 \\
406\end{array}$ & $\begin{array}{l}2175 \\
1504 \\
1761 \\
1244\end{array}$ & $\begin{array}{r}2060 \\
2178 \\
1565 \\
742\end{array}$ & $\begin{array}{l}2299 \\
1991 \\
1706 \\
1035\end{array}$ & $\begin{array}{r}2180 \\
2084 \\
1636 \\
888\end{array}$ \\
\hline $\begin{array}{l}\text { 2,4-D spray } \\
2,4-D \text { spray }\end{array}$ & $\begin{array}{l}1 \\
2\end{array}$ & $\begin{array}{l}4740 \\
4325\end{array}$ & $\begin{array}{l}2727 \\
2492\end{array}$ & $\begin{array}{l}1600 \\
3408\end{array}$ & $\begin{array}{l}2534 \\
2215\end{array}$ & $\begin{array}{l}1441 \\
1909\end{array}$ & $\begin{array}{l}1988 \\
2062\end{array}$ \\
\hline $\begin{array}{l}2,4,5-\mathrm{T} \text { spray } \\
2,4,5-\mathrm{T} \text { spray } \\
2,4,5-\mathrm{T} \text { spray } \\
2,4,5-\mathrm{T} \text { spray }\end{array}$ & $\begin{array}{l}0.5 \\
1 \\
2 \\
4\end{array}$ & $\begin{array}{l}4600 \\
4896 \\
3782 \\
3027\end{array}$ & $\begin{array}{l}2234 \\
2596 \\
1926 \\
2026\end{array}$ & $\begin{array}{l}3417 \\
3746 \\
2854 \\
2526\end{array}$ & $\begin{array}{l}1795 \\
2589 \\
2301 \\
1725\end{array}$ & $\begin{array}{l}1542 \\
1873 \\
1447 \\
1403\end{array}$ & $\begin{array}{l}1521 \\
2231 \\
1874 \\
1564\end{array}$ \\
\hline $\begin{array}{l}\text { Dicamba spray } \\
\text { Dicamba spray } \\
\text { Dicamba spray } \\
\text { Dicamba spray }\end{array}$ & $\begin{array}{l}0.5 \\
1 \\
2 \\
4\end{array}$ & $\begin{array}{l}4264 \\
4775 \\
3747 \\
3263\end{array}$ & $\begin{array}{l}2377 \\
2531 \\
1738 \\
1652\end{array}$ & $\begin{array}{l}3320 \\
3653 \\
2742 \\
2458\end{array}$ & $\begin{array}{l}2460 \\
1833 \\
2658 \\
2357\end{array}$ & $\begin{array}{l}1597 \\
1915 \\
1978 \\
1885\end{array}$ & $\begin{array}{l}2028 \\
1874 \\
2318 \\
2121\end{array}$ \\
\hline $\begin{array}{l}\text { Picloram granule } \\
\text { Picloram granule } \\
\text { Picloram granule }\end{array}$ & $\begin{array}{l}1 \\
2 \\
4\end{array}$ & $\begin{array}{l}2252 \\
2351 \\
2708\end{array}$ & $\begin{array}{l}1288 \\
1579 \\
1491\end{array}$ & $\begin{array}{l}1770 \\
1965 \\
2100\end{array}$ & $\begin{array}{r}1750 \\
1125 \\
437\end{array}$ & $\begin{array}{r}1540 \\
1892 \\
766\end{array}$ & $\begin{array}{r}1645 \\
1508 \\
602\end{array}$ \\
\hline $\begin{array}{l}\text { Dicamba granule } \\
\text { Dicamba granule } \\
\text { Dicamba granule }\end{array}$ & $\begin{array}{l}1 \\
2 \\
4\end{array}$ & $\begin{array}{l}4649 \\
3175 \\
3061\end{array}$ & $\begin{array}{l}2163 \\
2376 \\
1721\end{array}$ & $\begin{array}{l}3406 \\
2776 \\
2391\end{array}$ & $\begin{array}{l}1647 \\
2170 \\
1197\end{array}$ & $\begin{array}{l}2103 \\
2130 \\
1815\end{array}$ & $\begin{array}{l}1875 \\
2136 \\
1506\end{array}$ \\
\hline Mean & & 3409 & 1878 & 2644 & 1883 & 1714 & 1798 \\
\hline $\begin{array}{l}\text { significant differe } \\
\text { een varieties (mean } \\
\text { ng herbicides (mea } \\
\text { een varieties within } \\
\text { een herbicides with }\end{array}$ & $\begin{array}{l}\text { same herbicide } \\
\text { he same variety }\end{array}$ & $\begin{array}{r}\text { June } 10 \\
1252 \\
878 \\
1631 \\
1240\end{array}$ & $\begin{array}{c}\text { October } 7 \mathrm{~d} \\
280 \\
557 \\
154 \\
788\end{array}$ & & & & \\
\hline
\end{tabular}


Table 4. Oven-dry weight (lb/acre) of vegetation on a Common bermudagrass site near College Station, Texas, after application of herbicide sprays and granules at two dates. ${ }^{a}$

\begin{tabular}{|c|c|c|c|c|c|}
\hline \multirow[b]{2}{*}{ Chemical applied } & \multirow[b]{2}{*}{$\begin{array}{c}\text { Rate } \\
\text { (lb/acre) }\end{array}$} & \multicolumn{2}{|c|}{ Applied October 14, 1971} & \multicolumn{2}{|c|}{ Applied May 15,1972} \\
\hline & & $\begin{array}{c}\text { Harvested } \\
\text { June } 20,1972\end{array}$ & $\begin{array}{c}\text { Harvested } \\
\text { Nov. 9, } 1972\end{array}$ & $\begin{array}{c}\text { Harvested } \\
\text { June } 20,1972\end{array}$ & $\begin{array}{c}\text { Harvested } \\
\text { June } 9,1972\end{array}$ \\
\hline Untreated & & 1336 & 1869 & 1883 & $1589 \mathrm{c}$ \\
\hline Picloram spray & 0.25 & 1436 & 1488 & 1603 & $1608 \mathrm{c}$ \\
\hline Picloram spray & 0.5 & 1911 & 1788 & 1513 & 2412 e \\
\hline Picloram spray & 1 & 2086 & 2238 & 1338 & $2396 \mathrm{e}$ \\
\hline $2,4-D$ spray & 1 & 1854 & 1415 & 1890 & $1764 \mathrm{~cd}$ \\
\hline $2,4-D$ spray & 2 & 1193 & 1346 & 1454 & $2185 \mathrm{de}$ \\
\hline $2,4,5-\mathrm{T}$ spray & 1 & 1743 & 1420 & 1303 & $1845 \mathrm{~cd}$ \\
\hline $2,4,5-\mathrm{T}$ spray & 2 & 1388 & 1317 & 1462 & $1809 \mathrm{~cd}$ \\
\hline Dicamba spray & 0.5 & 1990 & 1531 & 1755 & $1911 \mathrm{cde}$ \\
\hline Dicamba spray & 1 & 1283 & 1297 & 1323 & $1834 \mathrm{~cd}$ \\
\hline Picloram granule & 1 & 1845 & 1740 & 1535 & $2210 \mathrm{de}$ \\
\hline Picloram granule & 2 & 2081 & 1664 & 1266 & $1714 \mathrm{~cd}$ \\
\hline Karbutilate granule & 1 & - & - & 1203 & $1879 \mathrm{~cd}$ \\
\hline Karbutilate granule & 2 & - & - & 1165 & $1080 \mathrm{~b}$ \\
\hline Karbutilate granule & 4 & - & - & 1100 & $410 \mathrm{a}$ \\
\hline
\end{tabular}

${ }^{a}$ Values followed by the same letter in the last column are not significantly different at the $5 \%$ level. Values in the first three columns are not significantly different at the $5 \%$ level.

month after treatment (data not shown). Degree of injury to bermudagrass depended directly upon application rate of picloram. Four $\mathrm{lb} / \mathrm{acre}$ of picloram reduced grass yield over $95 \%$ of that from untreated plots. Grass treated with picloram sprays of $0.5 \mathrm{lb} /$ acre was injured less than grass treated with higher picloram rates; but grass yield was significantly less than that from untreated plots. Sprays of 2-4-D, 2,4-5-T, and dicamba and granules of dicamba up to 4 $\mathrm{lb} /$ acre applied in May, 1971, did not significantly reduce yields of Common bermudagrass.

All herbicides applied in May, 1971, reduced oven-dry forage yields of Coastal and Coastcross-1 harvested 1 month after treatment, although results for 2,4-D,
2,4-5-T, and lower rates of dicamba on Coastcross-1 were not significantly different from those from untreated areas (Table 3). Reduction was greatest with highest rates of picloram. Five months after treatment, picloram spray and granular treatments at 2 and $4 \mathrm{lb} / \mathrm{acre}$ and dicamba granules at 1 and $4 \mathrm{lb} / \mathrm{acre}$, significantly reduced yield of Coastal bermudagrass. Granular formulations of picloram at $4 \mathrm{lb} /$ acre reduced yield of Coastcross-1. Forage yields from all other herbicide treatments were similar to untreated plots of Coastal and Coastcross- 1 bermudagrass. In 1971, forage yield of Coastal was consistently greater than that of Coastcross.

Yield of Common bermudagrass treated in October 1971 and harvested in
June and November, 1972, was not reduced by herbicide treatments (Table 4). Unlike results from picloram applied in October, 1970, picloram applications in October, 1971, at similar rates did not reduce grass yield. Reduced phytotoxicity of picloram applied in October, 1971, compared with that applied in October, 1970, is attributed to more abundant rainfall (Table 2), which may have leached toxic levels of picloram from that root zone and allowed more overall growth of grass. Grass yield tended to be highest on picloram-treated areas, probably because of the benefit of prolonged weed control.

No differences in bermudagrass yields in June, 1972, occurred among treatments applied to Common bermudagrass in May 1972 (Table 4). However, on the same plots in November, 1972, more forage was harvested where sprays of picloram at 0.5 and $1 \mathrm{lb} /$ acre, 2,4-D at 2 $\mathrm{lb} / \mathrm{acre}$, and picloram granules at $1 \mathrm{lb} / \mathrm{acre}$ had been applied than from other plots. Karbutilate at 2 and $4 \mathrm{lb} /$ acre reduced forage yields, but karbutilate at $1 \mathrm{lb} / \mathrm{acre}$ did not.

Fall applications of picloram, 2,4-D, 2,4,5-T, and dicamba in 1971 did not reduce forage yields of Coastal and Coastcross-1 bermudagrass harvested in June, 1972, or November, 1972 (Table 5). Coastal yield was greater in all picloram treatments in June, 1972, than in the untreated plots. Abundant rainfall apparently removed enough residual picloram to allow normal growth.

Except for karbutilate, which caused increased yield of Coastal in June, 1972,

Table 5. Oven-dry weight (lb/acre) of vegetation on Coastal and Coastcross-1 bermudagrass sites at College Station, Texas, after application of herbicide sprays and granules on October 14, 1971, and harvested at two dates.

\begin{tabular}{|c|c|c|c|c|c|c|c|}
\hline \multirow[b]{2}{*}{ Chemical applied } & \multirow{2}{*}{$\begin{array}{c}\text { Rate } \\
\text { (lb/acre) }\end{array}$} & \multicolumn{3}{|c|}{ Harvested June $20,1972^{1}$} & \multicolumn{3}{|c|}{ Harvested November $9,1972^{1}$} \\
\hline & & Coastal & Coastcross- 1 & $\overline{\text { Mean }}$ & Coastal & Coastcross-1 & Mean \\
\hline Untreated & & 537 & 772 & 654 & 1799 & 1848 & 1824 \\
\hline $\begin{array}{l}\text { Picloram spray } \\
\text { Picloram spray } \\
\text { Picloram spray }\end{array}$ & $\begin{array}{l}0.25 \\
0.5 \\
1\end{array}$ & $\begin{array}{r}888 \\
886 \\
1033\end{array}$ & $\begin{array}{r}1324 \\
720 \\
1002\end{array}$ & $\begin{array}{r}1106 \\
803 \\
1018\end{array}$ & $\begin{array}{l}1469 \\
1578 \\
1730\end{array}$ & $\begin{array}{l}1875 \\
1744 \\
1504\end{array}$ & $\begin{array}{l}1672 \\
1661 \\
1617\end{array}$ \\
\hline $\begin{array}{l}2,4-D \text { spray } \\
2,4-D \text { spray }\end{array}$ & $\begin{array}{l}1 \\
2\end{array}$ & $\begin{array}{l}567 \\
601\end{array}$ & $\begin{array}{l}501 \\
778\end{array}$ & $\begin{array}{l}534 \\
690\end{array}$ & $\begin{array}{l}1322 \\
1563\end{array}$ & $\begin{array}{l}1544 \\
1693\end{array}$ & $\begin{array}{l}1433 \\
1628\end{array}$ \\
\hline $\begin{array}{l}2,4,5-\mathrm{T} \text { spray } \\
2,4,5-\mathrm{T} \text { spray }\end{array}$ & $\begin{array}{l}1 \\
2\end{array}$ & $\begin{array}{l}542 \\
626\end{array}$ & $\begin{array}{l}771 \\
864\end{array}$ & $\begin{array}{l}656 \\
745\end{array}$ & $\begin{array}{l}1463 \\
1345\end{array}$ & $\begin{array}{l}1658 \\
1412\end{array}$ & $\begin{array}{l}1560 \\
1378\end{array}$ \\
\hline $\begin{array}{l}\text { Dicamba spray } \\
\text { Dicamba spray }\end{array}$ & $\begin{array}{l}0.5 \\
1\end{array}$ & $\begin{array}{l}715 \\
542\end{array}$ & $\begin{array}{l}834 \\
637\end{array}$ & $\begin{array}{l}774 \\
590\end{array}$ & $\begin{array}{l}1583 \\
1617\end{array}$ & $\begin{array}{l}1751 \\
1860\end{array}$ & $\begin{array}{l}1667 \\
1738\end{array}$ \\
\hline $\begin{array}{l}\text { Picloram granule } \\
\text { Picloram granule }\end{array}$ & $\begin{array}{l}1 \\
2\end{array}$ & $\begin{array}{l}889 \\
961\end{array}$ & $\begin{array}{r}1002 \\
803\end{array}$ & $\begin{array}{l}946 \\
882\end{array}$ & $\begin{array}{l}2013 \\
1428\end{array}$ & $\begin{array}{l}2065 \\
1399\end{array}$ & $\begin{array}{l}2039 \\
1414\end{array}$ \\
\hline Mean & & 732 & 834 & 783 & 1576 & 1696 & 1636 \\
\hline
\end{tabular}

\begin{tabular}{lcc}
\hline \hline${ }^{1}$ Least significant differences: & June 20 data & November 9 data \\
Between varieties (mean) & 189 & 633 \\
Among herbicides (mean) & 238 & 385 \\
Between varieties within the same herbicide & 359 & 767 \\
Between herbicides within the same variety & 336 & 546
\end{tabular}


Table 6. Oven-dry weight (lb/acre) of vegetation on Coastal and Coastcross-1 bermudagrass sites at College Station, Texas, after application of herbicide sprays and granules on May 15, 197.2, and harvested at two dates.

\begin{tabular}{|c|c|c|c|c|c|c|c|}
\hline \multirow[b]{2}{*}{ Chemical applied } & \multirow{2}{*}{$\begin{array}{c}\text { Rate } \\
\text { (lb/acre) }\end{array}$} & \multicolumn{3}{|c|}{ Harvested June $20,1972^{1}$} & \multicolumn{3}{|c|}{ Harvested November $9,1972^{1}$} \\
\hline & & Coastal & Coastcross-1 & Mean & Coastal & Coastcross-1 & Mean \\
\hline Untreated & & 537 & 772 & 654 & 1799 & 1848 & 1824 \\
\hline $\begin{array}{l}\text { Picloram spray } \\
\text { Picloram spray } \\
\text { Picloram spray }\end{array}$ & $\begin{array}{l}0.25 \\
0.5 \\
1\end{array}$ & $\begin{array}{l}766 \\
496 \\
734\end{array}$ & $\begin{array}{l}815 \\
705 \\
668\end{array}$ & $\begin{array}{l}790 \\
600 \\
701\end{array}$ & $\begin{array}{l}1632 \\
1778 \\
1747\end{array}$ & $\begin{array}{l}2425 \\
1735 \\
1618\end{array}$ & $\begin{array}{l}2028 \\
1756 \\
1682\end{array}$ \\
\hline $\begin{array}{l}\text { 2,4-D spray } \\
2,4-D \text { spray }\end{array}$ & $\begin{array}{l}1 \\
2\end{array}$ & $\begin{array}{l}709 \\
811\end{array}$ & $\begin{array}{l}878 \\
774\end{array}$ & $\begin{array}{l}794 \\
792\end{array}$ & $\begin{array}{l}1808 \\
1413\end{array}$ & $\begin{array}{l}2469 \\
1607\end{array}$ & $\begin{array}{l}2138 \\
1510\end{array}$ \\
\hline $\begin{array}{l}2,4,5-T \text { spray } \\
2,4,5-T \text { spray }\end{array}$ & $\begin{array}{l}1 \\
2\end{array}$ & $\begin{array}{l}579 \\
880\end{array}$ & $\begin{array}{l}910 \\
982\end{array}$ & $\begin{array}{l}744 \\
931\end{array}$ & $\begin{array}{l}1549 \\
1826\end{array}$ & $\begin{array}{l}2954 \\
2387\end{array}$ & $\begin{array}{l}2252 \\
2106\end{array}$ \\
\hline $\begin{array}{l}\text { Dicamba spray } \\
\text { Dicamba spray }\end{array}$ & $\begin{array}{l}0.5 \\
1\end{array}$ & $\begin{array}{l}750 \\
706\end{array}$ & $\begin{array}{l}886 \\
745\end{array}$ & $\begin{array}{l}818 \\
726\end{array}$ & $\begin{array}{l}1877 \\
1641\end{array}$ & $\begin{array}{l}1660 \\
2210\end{array}$ & $\begin{array}{l}1768 \\
1926\end{array}$ \\
\hline $\begin{array}{l}\text { Picloram granule } \\
\text { Picloram granule }\end{array}$ & $\begin{array}{l}1 \\
2\end{array}$ & $\begin{array}{l}588 \\
631\end{array}$ & $\begin{array}{l}704 \\
977\end{array}$ & $\begin{array}{l}646 \\
804\end{array}$ & $\begin{array}{l}1667 \\
1145\end{array}$ & $\begin{array}{l}1958 \\
1491\end{array}$ & $\begin{array}{l}1812 \\
1318\end{array}$ \\
\hline $\begin{array}{l}\text { Karbutilate granule } \\
\text { Karbutilate granule } \\
\text { Karbutilate granule }\end{array}$ & $\begin{array}{l}1 \\
2 \\
4\end{array}$ & $\begin{array}{r}1006 \\
729 \\
963\end{array}$ & $\begin{array}{l}718 \\
625 \\
506\end{array}$ & $\begin{array}{l}862 \\
677 \\
734\end{array}$ & $\begin{array}{l}1503 \\
1390 \\
1409\end{array}$ & $\begin{array}{l}1867 \\
1110 \\
1184\end{array}$ & $\begin{array}{l}1685 \\
1250 \\
1296\end{array}$ \\
\hline Mean & & 726 & 778 & 752 & 1612 & 1902 & 1757 \\
\hline
\end{tabular}

\begin{tabular}{lcc}
\hline 1Least significant differences: & June 20 data & November 9 data \\
Between varieties (mean) & 155 & 869 \\
Among herbicides (mean) & 279 & 555 \\
Between varieties within the same herbicide & 404 & 1078 \\
Between herbicides within the same variety & 395 & 786
\end{tabular}

herbicides applied in May, 1972, resulted in no significant differences in forage yield of Coastal or Coastcross-1 bermudagrass 1 and 6 months after treatment (Table 6). Karbutilate tended to reduce yield of Coastcross at 2 and $4 \mathrm{lb} /$ acre in plots harvested in the fall, but yield was not significantly different from that of the control. Growth of bermudagrass was much greater in the spring of 1971 than 1972 because of more rainfall and favorable temperature between time of treatment and harvest.

\section{Discussion}

Herbicides 2,4-D, 2,4,5-T, and dicamba usually did not reduce yield of established Common, Coastal, or Coastcross-1 bermudagrass, regardless of rate or time of treatment. The high rate of
2,4,5-T (4 lb/acre) in spring tended to discolor the grass, but injury was temporary. Picloram at 2 and $4 \mathrm{lb} /$ acre applied during periods of dry weather severely reduced bermudagrass growth. Most of the grass harvested in plots treated with picloram at $4 \mathrm{lb} /$ acre was Kleingrass, which was very resistant to picloram and other herbicides. In most weed and brush control programs, rate of herbicide would seldom exceed $1 \mathrm{lb} /$ acre. Picloram at rates of $1 \mathrm{lb} / \mathrm{acre}$ or below sometimes injured bermudagrass but reduced growth was temporary and regrowth sometimes exceeded that of the control, probably a result of weed control. However, in areas of limited rainfall or irrigation (below 20 inches annually), picloram may significantly reduce bermudagrass stand and production the first year after treatment if phytotoxic levels of picloram remain in the root zone. Common, Coastal and Coastross-1 varieties of bermudagrass responded similarly to each herbicide studied.

\section{Literature Cited}

Bovey, R. W., J. R. Baur, and H. L. Morton. 1969. Control of huisache and associated woody species in south Texas. J. Range Manage. 23:47-50.

McBee, G. G. 1966. Postemergence herbicides studies on certain weeds in bermudagrass turf. Tex. Agr. Exp. Sta. Progr. Rep. 2408. $5 \mathrm{p}$.

Meyer, R. E., and R. W. Bovey. 1973. Control of woody plants with herbicide mixtures. Weed Sci. $21: 423-426$.

Reber, L. J., R. K. Miller, J. A. Tweedy, and J. D. Butler. 1971. Herbicidal effects of picloram on bermudagrass. Weed Sci. 19:521-524.

Scifres, C. J., and G. O. Hoffman. 1972. Comparative susceptibility of honey mesquite to dicamba and 2,4,5-T. J. Range Manage. 25:143-146. 